\title{
CCD photometric study of the contact binary FG Hydrae
}

\author{
Y. Yang and Q. Liu \\ Yunnan Observatory, Chinese Academy of Sciences, Kunming, Yunnan Province, China \\ United Laboratory of Optical Astronomy, Chinese Academy of Sciences, China \\ National Astronomical Observatories, Chinese Academy of Sciences, China \\ e-mail: bily@public.km.yn.cn
}

Received October 21, 1999; accepted March 21, 2000

\begin{abstract}
A new light curve of the contact binary FG Hya is presented in this paper. The new light curve and those obtained by other authors from 1955 to 1985 show the change in the shape of the light curves of the system. The difference in the depths between the primary and secondary eclipses increased yearly from 1955 to 1985 , but the present light curve seems to exhibit the same shape as the light curve of Smith in 1955. The long-term decrease of the orbital period of the system may be clear if Mahdy et al.'s (1985) timings in question were counted out. The present light curve and those obtained by Binnendijk in 1962 and Yang et al. in 1982 are all analyzed by means of the latest version of the WD program. The results show that FG Hya is an over-contact binary with a small mass ratio. Although the light curves of the system changed considerably, the solutions of the light curves obtained in 1955, 1982 and 1999 are in agreement.
\end{abstract}

Key words: star — binary — light curve change — period change - photometric solutions

\section{Introduction}

The variable FG Hydrae $\left(\mathrm{BD}+03^{\circ} 1979\right)$ was discovered by Hoffmeister (1934). It was classified as a cluster type by Tsesevich (1949) by using visual observations. The first photoelectric photometric observations were obtained by Smith $(1955,1963)$, who discovered that the system belonged to an W Ursae Majoris type that undergoes complete eclipses. The complete light curves of the system were obtained by Binnendijk (1963), Yang et al. (1991) and Mahdy et al. (1985). According to Qian

Send offprint requests to: Y. Yang

* Table 4 is only available in electronic form at http://www.edpsciences.org et al. (1999), the orbital period of FG Hya underwent several sudden jumps in 1952, 1962, 1982, 1985 and 1989 and the light curves of the system also show the variable O'Connell effect. The photometric solutions were obtained by Mochnacki et al. (1972), Twigg (1979), Lafta et al. (1986), Jabbar et al. (1986) and Yang et al. (1991). Their studies indicate that the system is an A-type overcontact W UMa binary. Recently Wenxian Lu \& Slavek M. Rucinski (1999) published radial velocity studies for this binary. The external error of the derived radial velocities from their DDO observations is smaller than $1.8 \mathrm{~km} \mathrm{~s}^{-1}$. The derived mass ratio from their better phase covered radial velocity curve is $0.112(04)$ which is close to those from the photometric solutions presented by Mochnacki et al. (1972), Twigg (1979), Lafta et al. (1986), Jabbar et al. (1986) and Yang et al. (1991).

Because of the variable O'Connell effect seen in the light curve and the over-contact nature of the binary, FG Hya was included in the observing program of short period variables at the Yunnan Observatory using the 100-cm reflector telescope.

\section{Observations}

The observations of FG Hya in the $V$-band were carried out over 4 nights in March, 1999, with the PI1024 TKB CCD photometric system attached to the $100-\mathrm{cm}$ reflector telescope at the Yunnan Observatory in China. The effective field of view of the photometric system is 6.5 square arc minutes at the Cassegrain focus and its $B V$ color system approximates the standard Johnson $B V$ photometric system (Yang \& Li 1999). The coordinates of the comparison star and the check star used are listed in Table 1, respectively. The comparison star and the check star are so close to the variable that they are in the same field of the observation together with the program star.

The integration time for each image is $100 \mathrm{~s}$. A total of 207 images in $V$ band were obtained for 4 nights in March, 
Table 1. The coordinates of the variable, comparison star and check star

\begin{tabular}{lcc}
\hline star & R.A.(1999.0) & Dec.(1999.0) \\
\hline variable & $08^{\mathrm{h}} 27^{\mathrm{m}} 01^{\mathrm{s}}$ & $03^{\mathrm{o}} 31^{\prime} 02^{\prime \prime}$ \\
comparison star & 082657 & 033208 \\
check star & 082656 & 032946 \\
\hline
\end{tabular}

Table 2. The times of minimum light of FG Hya

\begin{tabular}{lccc}
\hline HJD 2451240+ & Min. & $(\mathrm{O}-\mathrm{C})_{1}$ & $(\mathrm{O}-\mathrm{C})_{2}$ \\
\hline $5.2079(4)$ & I & -0.0282 & -0.0007 \\
$6.1925(3)$ & I & -0.0261 & 0.0004 \\
$7.1762(9)$ & I & -0.0269 & 0.0006 \\
$8.1592(4)$ & I & -0.0274 & 0.0001 \\
\hline
\end{tabular}

1999. The aperture photometry package of IRAF was used to reduce the images. The reduced results show that the difference between the magnitude of the check star and that of the comparison star is constant within probable error of \pm 0.006 magnitude. Extinction corrections were not made since the comparison star was so close to the variable.

From the observations, four times of minimum light were derived using parabola fitting. The new times of the minimum light are listed in Table 2 , in which the $(\mathrm{O}-\mathrm{C})_{1}$ values are calculated by means of the light element formula given by Smith (1963)

$$
\text { Min.I = HJD 2436968.7067 + 0. } 32783433 E .
$$

From 36 photoelectric times of minimum light collected in the references and the new ones in the present article, the $(\mathrm{O}-\mathrm{C})$ values of the minima computed with the above ephemeris are plotted in Fig. 1. This diagram shows that several sudden jumps in the orbital period of FG Hya may occur. The timings of Mahdy et al. (1985) may be crucial in the $\mathrm{O}-\mathrm{C}$ diagram (see Fig. 1). When their observations were checked, one of their timings (the first one listed) was found to be incorrect. They gave -0.0017 for the $\mathrm{O}-\mathrm{C}$ whereas the calculated $\mathrm{O}-\mathrm{C}$ is -0.031 ! Their other values are correct as given. But the question remains, their light curves appear to be distorted by heavy and asymmetrical spot(s). Therefore, the timings from the most extreme light curves could be suspected to be off because of the substantial spot(s). If the timings near epoch 28000 from Mahdy et al.'s observations are not taken into account, one can see clearly that the period of FG Hya was decreasing (see Fig. 2) and then a parabola ephemeris can be derived as the following:

Min.I=HJD 2436968.7345+0 ${ }^{\mathrm{d}} 32783966 E-1.410^{-10} E^{2} .(2)$

The period change rate of FG Hya is $\delta p / p=-2.810^{-10}$. However, since 1989 the period has been quite stable. From our times and the ones after 1989, a new linear

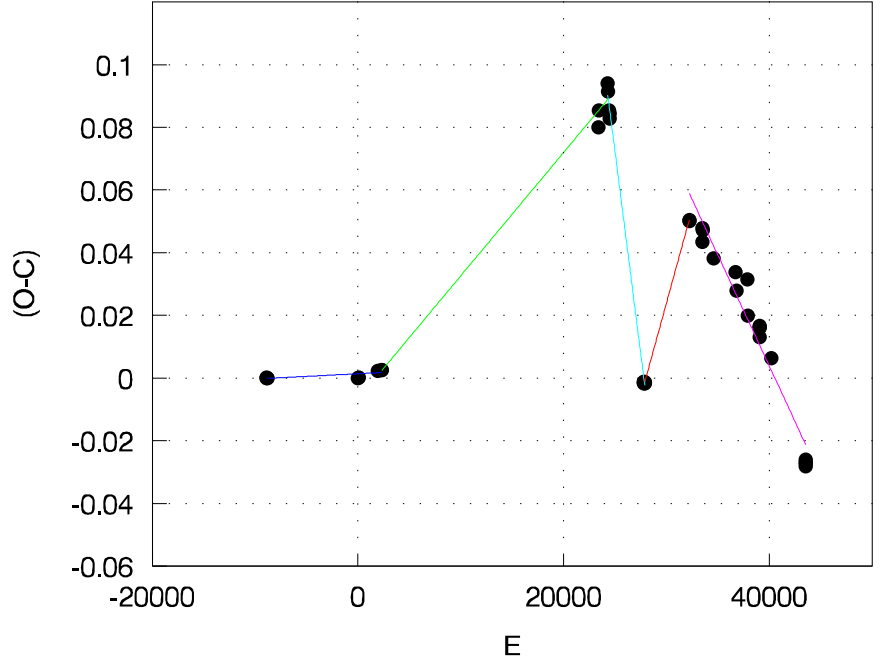

Fig. 1. The sudden changes in the orbital period of FG Hya

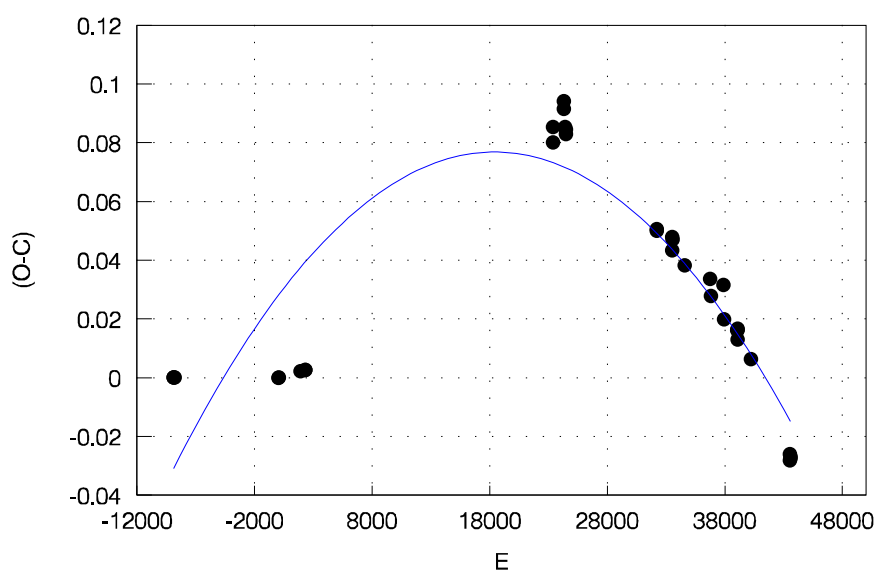

Fig. 2. Lone-term decrease of the orbital period for FG Hya

ephemeris, more consistent with the period from 1989 to 1999, was derived:

Min.I = HJD 2451248.1591(6) + 0.32782743(20)E,

which was used to compute the phases of our observations and the $(\mathrm{O}-\mathrm{C})_{2}$ values in Table 2 .

A total of 207 individual observations have been obtained and listed in Table 4 with their Heliocentric Julian Day, phases and magnitude differences between the variable and the comparison star. The light curve of the system is shown as the solid circle points in Fig. 3. Since the period of FG Hya is close to one third of a day, it is difficult to obtain a complete light curve in a season. Most of the phases of the present light curve were covered in the four observing sessions, but an interval of 0.1 in phase (from $0.35 \mathrm{p}$ to $0.45 \mathrm{p}$ ) is still is not covered.

As noted by Binnendijk (1963), the shape of the light curve of the system was changing. A graphical depiction of the variable shape of the light curves in $V$ band is shown in Fig. 4, which gives the observations carried out by Smith 


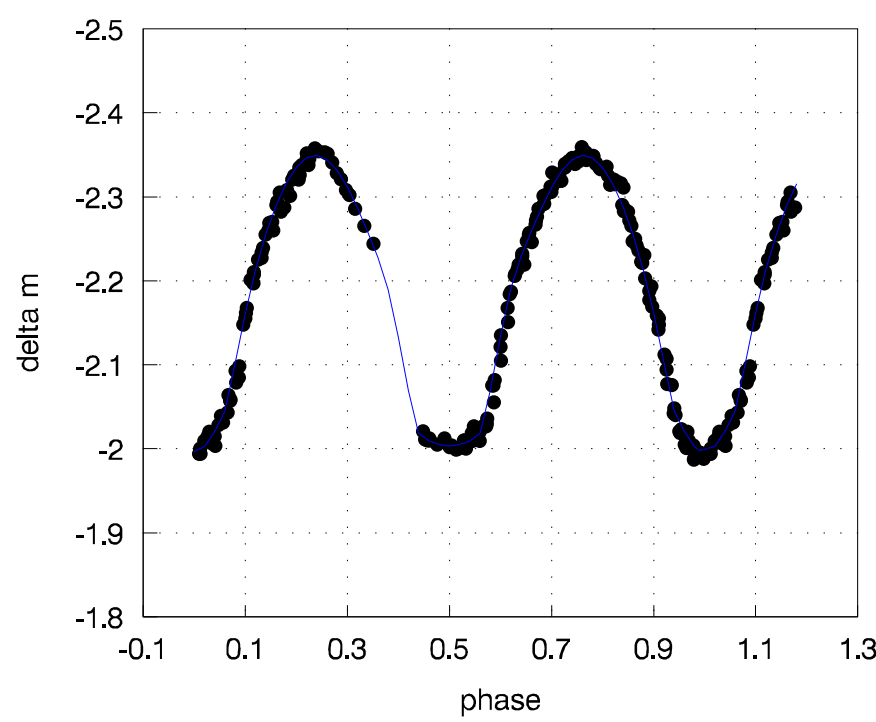

Fig. 3. The light curve of FG Hya. The solid circle points indicate the observations and the line shows those from the model (see the text)

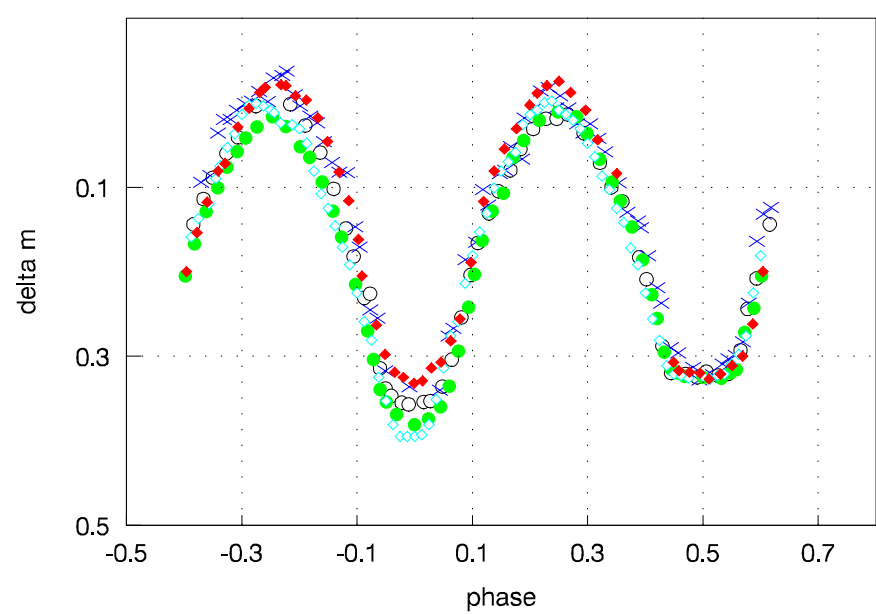

Fig. 4. The variation of the lignt curves of FG Hya. The explanation of the different synbols used can be seen in the text

(X's) in 1955, by Binnendijk (open circles) in 1961-62, by Yang et al. (solid circles) in 1982, by Mahdy et al. (open diamonds) in 1985 and by the present authors (solid diamonds) in 1999. For comparison, all light curves are reduced assuming that they could have the same light level at the phase of 0.5. This level was chosen to normalize all of the light curves to simply demonstrate the substantial variability of the light curve over time. As shown in Fig. 4, the variation of the light curve of the system is very obvious. Some parameters of the light curves for FG Hya are listed in Table 3, from which one can see that the difference of the eclipsing depth between the primary eclipse and the secondary one is considerably changing while neither the O'Connell effect (two maxima in the light curves are unequal), nor its variation is obvious.
Table 3. The parameters of the $V$ light curve properties of FG Hya

\begin{tabular}{lrrrrr}
\hline obs. date & 1955 & $1961-62$ & 1982 & 1985 & 1999 \\
\hline Max.I-Min.I & -0.350 & -0.339 & -0.369 & -0.397 & -0.357 \\
Max.I-Min.II & -0.333 & -0.310 & -0.314 & -0.327 & -0.350 \\
Max.II-Min.I & -0.372 & -0.352 & -0.363 & -0.394 & -0.353 \\
Max.II-Min.II & -0.355 & -0.323 & -0.314 & -0.324 & -0.346 \\
Max.I-Max.II & 0.022 & 0.013 & -0.006 & -0.003 & -0.004 \\
Min.I-Min.II & 0.017 & 0.029 & 0.055 & 0.070 & 0.007 \\
\hline
\end{tabular}

\section{Photometric solutions}

Photometric solutions were obtained by means of the WD program which includes a new reflection treatment, the option of using non-linear limb-darkening laws and the ability to adjust spot parameters. All the observations were used in computing the solutions. The convergence of the minimization procedure was obtained by means of the method of multiple subsets (Wilson \& Biermann 1976).

The adopted parameters in the solutions are described as follows: a temperature of $5900 \mathrm{~K}$ for Star 1 (the star eclipsed at Min.I), Claret et al.'s (1990) values of the limb darkening coefficient $\left(x_{1}=x_{2}=0.640\right)$, Lucy's (1967) values of the gravity darkening coefficients $\left(g_{1}=g_{2}=0.320\right)$ and Rucinski's (1969) values of the albedo $\left(A_{1}=A_{2}=\right.$ 0.500), which corresponds to the spectral type of G0 for FG Hya. The adjustable parameters were the orbital inclination, $i$, the mean temperature of Star $2, T_{2}$, the potential of the components, $\Omega_{1}$ and $\Omega_{2}$, and the monochromatic luminosity of Star $1, L_{1}$ (the Planck function was used in computing the luminosity).

Solutions were made for a series of fixed values of the mass ratio $q=m_{2} / m_{1}(0.10,0.15,0.20,0.30,0.40,0.50$, $0.60,0.70,0.80,0.90,1.50,2.00,2.50,3.00,4.00,5.00$ and $6.00)$. Assuming that initially it was a detached system, the differential corrections started from mode 2, but the converged solutions were always obtained at the contact mode 3 . The resulting sum $\Sigma$ of the square deviations of the converged solutions for each value of $q$ indicates that the fitting is best for $q=0.15$. At this point, the set of the adjustable parameters was expanded to include $q$. The mass ratio converged to a value of $q=0.1289$ in the final solution. This solution indicates that FG Hya is an A-type W UMa binary, in agreement with the results published by Mochnacki \& Doughty (1972), Twigg (1979), Lafta \& Grainger (1986), Jabbar et al. (1986) and Yang et al. (1991). The photometric parameters are listed in Table 5, where Star 1 indicates a more massive component and Star 2 is one of less mass. The computed light curve by using the parameters in Table 5 is shown as the solid line in Fig. 3. As shown in Fig. 3, the fit between the theoretical light curve and the observations is very good.

In order to compare the present solution with the previous ones, the light curves in $B$ and $V$ bands obtained 
Table 5. The photometric solutions of FG Hya

\begin{tabular}{|c|c|c|c|c|c|c|c|c|c|c|c|}
\hline \multicolumn{2}{|c|}{ parameters } & \multicolumn{2}{|c|}{ 1962's obs. } & \multicolumn{6}{|c|}{ 1982's obs. } & \multicolumn{2}{|r|}{ 1999's obs. } \\
\hline \multicolumn{2}{|l|}{ color } & \multicolumn{2}{|c|}{$V$} & \multicolumn{2}{|c|}{$B$} & \multicolumn{2}{|c|}{$V$} & \multicolumn{2}{|r|}{$B$} & & $V$ \\
\hline \multicolumn{2}{|c|}{$L_{1} /\left(L_{1}+L_{2}\right)$} & \multicolumn{2}{|c|}{$0.8621 \pm 0.0008$} & \multicolumn{2}{|c|}{$0.8637 \pm 0.0010$} & \multicolumn{2}{|c|}{$0.8636 \pm 0.0009$} & \multicolumn{2}{|c|}{$0.8616 \pm 0.0011$} & 0.864 & $48 \pm 0.0 .0007$ \\
\hline \multirow{2}{*}{\multicolumn{2}{|c|}{$\begin{array}{l}x_{1}=x_{2} \\
q=m_{2} / m_{1}\end{array}$}} & \multicolumn{2}{|c|}{0.640} & \multicolumn{2}{|c|}{0.780} & \multicolumn{2}{|c|}{0.640} & \multicolumn{2}{|c|}{0.780} & & 0.640 \\
\hline & & \multicolumn{2}{|c|}{$0.1306 \pm 0.0010$} & & & \multicolumn{2}{|c|}{$0.1273 \pm 0.0008$} & & & 0.128 & $889 \pm 0.0009$ \\
\hline & \multicolumn{2}{|c|}{$86.60 \pm 0.86$} & & & \multicolumn{2}{|c|}{$87.12 \pm 0.63$} & & & & $6.78 \pm 0.52$ \\
\hline \multicolumn{2}{|c|}{$A_{1}=A_{2}$} & \multicolumn{2}{|c|}{0.500} & & & \multicolumn{2}{|c|}{0.500} & & & & 0.500 \\
\hline \multicolumn{2}{|c|}{$g_{1}=g_{2}$} & \multicolumn{2}{|c|}{0.320} & & & \multicolumn{2}{|c|}{0.320} & & & & 0.320 \\
\hline$\Omega$ & & \multicolumn{2}{|c|}{$1.9908 \pm 0.0026$} & & & \multicolumn{2}{|c|}{$1.9834 \pm 0.0022$} & & & 1.98 & $821 \pm 0.0026$ \\
\hline$f$ & & \multicolumn{2}{|c|}{0.69} & & & \multicolumn{2}{|c|}{0.72} & & & & 0.74 \\
\hline$\Sigma$ & & \multicolumn{2}{|c|}{0.005321} & & & 0.0 & 04657 & & & & 0.003768 \\
\hline nponent & & 1 & & 2 & & & 2 & & 1 & & 2 \\
\hline K) & & 5900 & 583 & \pm 16 & 59 & & $5811=$ & $=18$ & 5900 & & $5852 \pm 19$ \\
\hline ole) & 0.527 & $7 \pm 0.0007$ & 0.2182 & \pm 0.0009 & $0.5335=$ & 0.0005 & $0.2217 \pm$ & 0.0006 & $0.5380 \pm$ & 0.0006 & $0.2179 \pm 0.000$ \\
\hline ide) & 0.587 & $2 \pm 0.0011$ & 0.2286 & \pm 0.0010 & $0.5963=$ & 0.0008 & $0.2332 \pm$ & 0.0007 & $0.6031 \pm$ & 0.0008 & $0.2293 \pm 0.0009$ \\
\hline ack) & 0.610 & \pm 0.0013 & 0.2762 & \pm 0.0025 & $0.6202=$ & 0.0009 & $0.2914 \pm$ & 0.0022 & $0.6265 \pm$ & 0.0018 & $0.2893 \pm 0.0025$ \\
\hline
\end{tabular}

by Binnendijk (1963) in 1961-62 and Yang et al. (1991) in 1982 were also re-analyzed in the same way. The results are also listed in Table 5, from which one can see that the solutions obtained from different observations are in agreement with each other despite the considerable change in the shape of the light curves.

We tried also to analyze the light curves obtained by Mahdy et al. (1985), but a reasonable solution could not be found. In the course of finding a solution by means of the DC program of the WD code, we found all obtained converged solutions difficult to interpret. The solutions indicated either orbital inclinations that were too small or mass ratios substantially larger than the spectroscopically determined one. When spot models were attempted, the solutions indicated that FG Hya would have to be classified as a W-type system. It is possible that FG Hya is an example of a small mass ratio binary that fluctuates between the $\mathrm{A}$ - and $\mathrm{W}$-type configuration, depending upon its current state of spot coverage. This is certainly not without precedence, as the similar system V677 Cen (Kilmartin et al. 1987) was found to possess equal temperatures for both components, i.e., on the boundary between A-type and W-type. Other W UMa binaries (e.g., TZ Boo, VW Cep) have displayed significantly changing depths of eclipses such that the normally less deep secondary eclipse was sometimes deeper than the primary one. Therefore we abandoned our attempt to find a final solution from Mahdy et al.'s observations. We suspect that either their observations are in question or the distortion of the light curves is so much because of the substantial spot that it is difficult to find a true solution.

\section{Conclusions and discussions}

FG Hya is an important and interesting binary, mostly because of its variable light curve and total eclipses. The totality of its eclipses allows it to have a photometrical determined mass ratio that is believable. In the present solutions, the obtained mass ratio of FG Hya is 0.1306 for the observations in 1962 (Binnendijk 1963), 0.1273 for the observations in 1882 (Yang et al. 1991) and 0.1289 for the present observations, respectively. The obtained values of the mass ratio of this binary are in agreement with each other and close to the derived one from the radial velocity curve (Lu et al. 1999).

The shape of the light curves of FG Hya has displayed considerable changes. From Fig. 4 and Table 3 one can see that the difference of the eclipsing depth between the primary eclipse and the secondary one considerably changes. From 1955 to 1985 the difference of the eclipsing depth increased yearly, but the present light curve seems to be similar in shape to that obtained by Smith in 1955. The variability of the light curve is probably due to varying starspots on one or probably both of the components because of the late spectral type of this system. In our solutions, spot modeling was not tried because of some uncertainty of spot parameters derived by the present model. What this system really needs is more complete coverage throughout a season, i.e., several light curves in one season, each one obtained over a week or two, in order to study monthly changes in the light curves, and then model each light curve with spots. If enough light curves are accumulated with standardized methods and equipment, a fiducial "spotless" curve should emerge (the upper envelope of all of the phased light curves taken, say, over a decade).

This system is important for evolutionary reasons as well. Its small mass ratio, over-contact nature and 
decreasing period would seem to indicate that it may be well on its way to coalescing. According to Maceroni \& van't Veer (1996), the mass of the secondary component of the system is only $0.15 m_{\odot}$ and the over-contact degree of the system is close to $80 \%$ which is in agreement with the present solution. Therefore, the secondary component could be unstable in both thermodynamics and dynamics and the instability of the secondary component could be responsible for the perplexing changes in both the light curve and the orbital period of the system. However, the light curve changes of FG Hya are more likely to be caused by varying starspots on one or probably both of the components because of their late-type spectra.

None the less, there are $9 \mathrm{~W}$ UMa type binaries with a smaller mass ratio than 0.2 (V677 Cen, FG Hya, XY Boo, AW UMa, TV Mus, RR Cen, MW Pav, $\epsilon$ CrA and EF Dra). These systems may be specifically important for studying structure and evolution of a binary. The evolution of these systems is controlled by the primary but the radius of the secondary is controlled by its contact with the primary. The small mass secondary has the large radius not resulting from any evolution of reactions in the core of the secondary and then it could be extremely heated by radiation from the primary. In addition, the secondary of these systems also receives energy through energy transfer from the primary to the secondary. Therefore, the structure and evolution of the secondary of these binaries should be very interesting. Some perplexing phenomena for these binaries could be observed. A similar binary, for example, AW UMa is an A-subtype W UMa binary with 0.080 of a mass ratio. Besides the variable light curve and decreasing period like that of FG Hya, secondary minima of AW UMa are systematically delayed with respect to the expected position in phase 0.5 given by primary minima (Pribulla et al. 1999). In any case, it may be interesting systematically to observe this group of the contact systems with a small mass ratio.
Acknowledgements. The authors would like to thank Dr. S. Qian and Dr. F. Li for their assistance in the observations and thank the unknown referee for his useful comments and improvement of the authors' English. The authors would also like to express their gratitude for the support from the Chinese National Science Foundation Committee and the Chinese Academy of Sciences.

\section{References}

Binnendijk L., 1963, AJ 68, 30

Claret A., Gimenez A., 1990, A\&A 230, 412

Hoffmeister C., 1934, Astron. Nachr. 253, 199

Jabbar S.R., et al., 1986, APSS 123, 325

Kilmartin P.M., Bradstreet D.H., Koch R.H., 1987, ApJ 319, 334

Lafta S.J., Grainger J.F., 1986, APSS 127, 153

Lu W., Rucinski S.M., 1999, AJ 118, 515

Lucy L.B., 1967, Zert. Astrophys. 65, 89

Maceroni C., van't Veer F., 1996, A\&A 311, 523

Mahdy H.A., Hamdy M.A., Soliman M.A., 1985, IBVS 2811

Mochnacki S.W., Doughty N.A., 1972, MNRAS 156, 243

Pribulla T., Chochol D., Rovithis-Livaniou H., Rovithis P., 1999, A\&A 345, 137

Qian S., Liu Q., Yang, Y., 1999, A\&A 341, 799

Rucinski S.M., 1969, A\&A 19, 245

Smith H.J., 1955, AJ 60, 179

Smith H.J., 1963, AJ 68, 39

Tsesevich V.P., 1949, Peremennye Zvezdy 7, 8

Twigg L.W., 1979, MNRAS 189, 907

Wilson R.E., Biermann, 1976, A\&A 48, 349

Yang Y., Liu Q., Zhang Y., Wang B., Lu L., 1991, Acta Astron. Sin. 32, 332

Yang Y., Li L., 1999, Publ. Yunnan Obs., No. 1, p. 32 